\title{
KLENOT - Near Earth and other unusual objects observations
}

\author{
Michal Kočer, Jana Tichá and Miloš Tichý \\ Kleť Observatory, Zátkovo nábř. 4, CZ-37001 České Budějovice, Czech Republic \\ email: klet@klet.cz
}

\begin{abstract}
The KLENOT project is a project of the Klet Observatory, Czech Republic, devoted to astrometric observations of Near-Earth objects, distant objects and comets. The improved effort of the large NEO surveys resulting in an increasing number of newly discovered NEOs calls for continuous follow-up astrometry to secure an accurate orbit determination of discovered bodies first in discovery opposition and then during next apparitions. Considering this urgent need of astrometric follow-up, the fact that many of these targets are fainter then magnitude $20.0 \mathrm{~V}$ and our results and experience in minor planet and comet CCD astrometry done at Klet since 1993, we decided to bring into operation a new 1-m class facility working on a permanent basis - the KLENOT telescope. The regular observing of the telescope started in March 2002 (the MPC code 246). Beside methods and techniques we use for follow-up astrometry we present most important results of the project.
\end{abstract}

Keywords. Minor planets, asteroids

\section{Introduction}

The discovery of near-Earth objects (NEOs) is an important contribution, but it would mean little without follow-up observations used to refine orbits and to secure an identification of a possible impact in the future. This need for astrometric follow-up of NEOs increases with increasing capabilities of the existing surveys and a growing interest towards smaller objects. This is why the KLENOT (KLEt observatory Near-Earth and Other unusual objects observations Team and Telescope) project has been established to provide follow-up astrometry of NEOs up to magnitude limit $\mathrm{m}_{\mathrm{V}}=22.0^{\mathrm{m}}$.

\section{The KLENOT telescope}

The KLENOT telescope was built using an existing dome and infrastructure of the Klet Observatory. The original mounting was upgraded and the optoelectronical control system was added. A new control and computer room was built.

Optical system: The KLENOT telescope has a $1.06-\mathrm{m}$ primary mirror and a primary focus corrector. The main f/3.0 mirror was fabricated by Carl Zeiss Jena using a Sital glass (Zerodur type). The primary focus corrector was computed by Sincon, Turnov, Czech Republic, and was fabricated by the Optical Facility of Charles University, Prague, Czech Republic, led by Jindřich Walter. The corrector contains four spherical lenses. The optical configuration is $\mathrm{f} / 2.7$ at the folded prime focus where the CCD camera is located.

$C C D$ camera: The CCD camera used for The KLENOT Project is CCD camera Photometrics Series 300 . The CCD chip sensor is SITe SI003B with format $1024 \times 1024$ pixels, pixel size $24 \times 24$ microns, and is back illuminated with high quantum efficiency; Q.E. $>80 \%$ in range $5500-8000 \AA$. Imaging array size is $24.6 \times 24.6$ millimeters. The field of view of the KLENOT Telescope is $33 \times 33$ arcminutes using the CCD camera mentioned above. The image scale is 1.9 arcseconds per pixel. The limiting magnitude is $\mathrm{m}_{\mathrm{V}}=21.5^{m}$ for 120 -sec exposure time in standard weather condition. 


\section{The KLENOT Project}

Confirmatory observations of newly discovered fainter NEO candidates: Some of new search facilities produce discoveries fainter than $\mathrm{m}_{\mathrm{V}}=20^{m}$ which need a larger telescope for confirmation and early follow-up. A 1-m class telescope is also very suitable for confirmation of very fast moving objects and our larger FOV enables to search for NEO candidates having a larger ephemeris uncertainty.

Follow-up astrometry of poorly observed NEOs: It is necessary to observe newly discovered NEOs in a longer arc during the discovery opposition when they get fainter. Special attention is given to "Virtual Impactors" and PHAs, target of future space missions or radar observations. On the other hand, it is necessary to find and use an optimal observing strategy to maximize orbit improvement of each asteroid.

Recoveries of NEOs in the second opposition: For the determination of reliable orbits it is required to observe asteroids in more then one opposition. If the observed arc in a discovery apparition is long enough, the chance for a recovery in the next apparition is good. If the observed arc at single opposition is not so good, we plan to search along the line of variation. For this purpose a larger field of view is an advantage.

Follow-up astrometry of other unusual objects: We plan to make follow-up astrometry of other unusual objects, i.e. Centaurs and transneptunian objects, both in discovery opposition and next apparitions. To obtain positions of brighter transneptunians, we propose to use longer exposures with magnitude limit about $\mathrm{m}_{\mathrm{V}}=22^{m}$. Considering the problem with securing adequate data for orbit computation of these objects, follow-up astrometry, at least of some of them, will be useful.

Cometary features: The majority of new ground-based discoveries of comets comes from large surveys devoted, predominantly, on Near Earth Asteroids. The first step in distinguishing these newly discovered members of the population of cometary bodies consists in confirmatory astrometric observations along with detection of their cometary features. A timely recognition of cometary features of a particular body having an unusual orbit can help in planning further observing campaigns.

Search for new asteroids: Our primary goal is astrometric follow-up of NEOs and other unusual objects. Moreover, all CCD images are processed not only for target objects, but also tested for possible new object(s). Obtained images will be processed with special reference to fast moving objects and slow moving objects.

Follow-up of Gamma-ray burst (GRB) optical counterparts: A part of observing time is devoted to GRB optical follow-up observations as a target of opportunity.

\section{Technology}

A special software package has been developed for the KLENOT Project at Klet using a combination of programs running on Windows and Linux platforms. The system consists of observation planning tools, data-acquisition, camera control and data processing tools. So, all the software mentioned bellow has been developed by KLENOT team.

Observation planning: The SQL database holds information on minor planets updated on daily bases from text-based databases; the MPC Orbit Database (MPCORB), maintained by the Minor Planet Center, and from the Asteroid Orbital Elements Database (ASTORB), created and maintained by E. Bowell at the Lowell Observatory. The asteroids listed on Spaceguard system Priority List and objects listed as a Virtual Impactors by SENTRY (JPL) or by CLOMON (NEODyS) are flagged in the SQL database as well. Besides the information on asteroids the database holds orbital elements and other useful data of all solar system objects discovered at Klet (database K_KLET) and also information on comets (database COMETS) created and updated from several sources by Klet. Also positions, times and observed objects on all of the processed plates and CCD images are 
stored in the database (CCD). For observation planning a web-based tool called ephem is used. The tool allows an observer to get ephemerides for one or more minor planets in a specified field in the sky at given time. The objects in the output list can be reduced to objects of given magnitude and/or type; i.e. to Near-Earth asteroids (NEAs), potentially hazardous asteroids (PHAs), Virtual Impactors (VI), Klet discoveries, critical list objects, unusual or distant minor planets, Trojans, Spaceguard Priority List objects and comets. The output list includes, beside designation, position in the sky, magnitude,and other usual ephemeris data, also information on object type, ephemeris uncertainty, date of last observation, length of orbital arc used in orbit computation. The output list is ordered by right ascension. Another program KAC - Klet' Atlas Coeli — shows stars and solar system objects with the line showing their daily motion on the sky on selected region in the sky. The size of the region corresponds to the FOV of the telescope used so it is also used to check the telescope position during observation. As a source of positions and magnitudes of stars the USNO-A2.0 star catalog is used.

Data acquisition and camera control: The CCD camera is controled by $\mathrm{V}++$ scripts. For exposure control and data-acquisition a set of scripts in VPascal (build-in programming language in $\mathrm{V}++$ ) has been written. The scripts store a sequence of several CCD frames (images) in one file in TIFF format. In the header of the sequence file information about the number of frames in the sequence, time, exposure time, equipment used and other information are included.

Data processing: The program Astrometry has been developed for the reduction of CCD images and identification of stars with USNO-A2.0 catalogue. Images taken by CCD camera are reduced and all objects with realized condition for signal to noise ratio are found on the image. These object are then identified with stars from the star catalogue. Equatorial coordinates of objects are then determined, at the same time stars with residuals greater than 1 arcsecond are excluded automatically and/or manually and magnitude of the objects is determined. The user then selects which object on the image the output should be made from. The output is directly in the MPC format. The time of observation and other information needed for the output are derived from the data stored in the header of the image file. Information about the processed CCD image (time, filename, frame number, equatorial coordinates of the center of the frame, telescope used, exposure time, position of objects on frame, etc.) are added into the SQL database of processed CCD images.

The measured astrometric position is checked before providing them to the community. Computing of residuals, i.e. differences between calculated and observed astrometric positions, is used for such proof (program residua). The calculation of residuals is based on osculating elements of the object near the current epoch, so they are acceptable mainly for the evaluation of actual observation data. Alongside the $\Delta-\mathrm{T}$ variation of the mean anomaly is determined. Checking of both residuals and the $\Delta-\mathrm{T}$ variation in mean anomaly helps in object identification. The next utility of the KLENOT's software package (program orbit) allows us a computation of preliminary orbital elements from observations for one or two nights on the assumption that an object is in perihelion, has been also developed. From observations over several nights the preliminary orbit of a new minor planet should be determined using Lagrange-Gauss method improved by variation of geocentric distances and variation of elements. Orbital elements of new discovered objects are stored in K_KLET database. We are working now on an improvement of this software for orbit determination taking into account perturbating mutual gravity within N-body system. For exact determination of planetary position the system uses The Planetary and Lunar Ephemerides DE405 provided by JPL. 


\begin{tabular}{|c|c|c|c|c|c|c|}
\hline | & \multicolumn{3}{|c|}{ asteroids } & \multicolumn{3}{|c|}{ NEA families } \\
\hline | year $\mid$ & all & NEA & PHA | & Apollo | & $\mathrm{Amc}$ & Aten \\
\hline | 2002 & 6324 & 2305 & 625 & 2305 & 963 & 205 \\
\hline 2003 & 12626 & 3603 & 831 & 3603 & 1953 & 304 \\
\hline 2004 & 3285 & 1183 & 224 & 1183 & 528 & 90 \\
\hline
\end{tabular}

Table 1. Total number of observations per year, total number of observation of NEAs and of the major NEA families obtained by KLENOT in the given year (by 2004 Aug.14)

\begin{tabular}{|l||c|c|c||c|c|c|}
\hline \multicolumn{4}{|c||}{ NEA } & \multicolumn{2}{c|}{ PHA } \\
\hline year & all & follow-up & early follow-up & all & follow-up & early follow-up \\
\hline 2002 & 377 & 213 & 145 & 75 & 47 & 23 \\
2003 & 438 & 242 & 215 & 84 & 48 & 25 \\
2004 & 298 & 151 & 95 & 56 & 24 & 10 \\
\hline
\end{tabular}

Table 2. Contribution of KLENOT project to observation of newly discovered NEAs and PHAs in given years (by 2004Aug.14). Legend: all — total number of world-wide discovered bodies; early follow-up - total number of bodies observed by KLENOT in early follow-up, i.e. the KLENOT's observation was published in discovery circular of given body; follow-up - total number of bodies observed by KLENOT in a discovery opposition

\section{Results}

The regular observations of the KLENOT project started in March 2002. By 2004 Aug. 1422.235 astrometric positions of Solar System Objects have been obtained; 7091 of them have been observations of NEAs (599 Atens, 3647 Apollos, 2845 Amors) and 755 have been observations of comets. Table 1 shows the the total number of observations and number of observation of NEAs/PHAs obtained by KLENOT and Table 2 shows the yearly contribution of KLENOT project to observation of newly discovered NEAs and PHAs in early follow-up as well as in later follow-up observation. Other important results of the KLENOT project are recoveries of 23 NEAs, recoveries of comets $\mathrm{C} / 2003$ A1, C/2003 A2, 100P and a discovery of Apollo-type asteroid 2003 LK and Aten-type asteroid 2003 UT55. Results obtained by the KLENOT team and telescope during its operation period show that this facility dedicated for permanent follow-up astrometry can significantly help in tracking NEAs worldwide.

\section{Acknowledgements}

This work has been sponsored by The Grant Agency of the Czech Republic Reg. number 205/98/0266, The 2000 NEO Shoemaker Grant of The Planetary Society, and The Grant Agency of the Czech Republic Reg. No. 205/02/P114.

\section{References}

Tichá, J., Tichý, M., and Moravec, Z. 2000a, Planet. Space Sci. 48, 787

Tichá, J., Tichý, M., and Moravec, Z. 2000b, Planet. Space Sci., 48, 955

Tichá, J., Tichý, M., and Moravec, Z. 2000c, in: A. Fitzsimmons, D. Jewitt, R. M. West (eds.), Minor Bodies in the Outer Solar System, Springer, p 165

Tichá, J., Tichý, M., and Kočer, M. 2002, ESA SP-500: ACM 2002, 793

Spahr, T.B. 2002, Minor Planet Electronic Circular, 2002-L05.

Sekanina, Z., Chodas, P. W., Tichý, M., Tichá, J., and Kočer, M. 2003, Astrophys. J., 591, L67 